\title{
Early post-treatment risk stratification of differentiated thyroid cancer: comparison of three high-sensitive Tg assays
}

\author{
Luca Giovanella ${ }^{1,2}$, Mauro Imperiali' ${ }^{2}$, Frederik A Verburg ${ }^{3}$ and Pierpaolo Trimboli1 \\ ${ }^{1}$ Department of Nuclear Medicine and Thyroid Centre, Oncology Institute of Southern Switzerland, Bellinzona, \\ Switzerland, ${ }^{2}$ Department of Laboratory Medicine, Ente Ospedaliero Cantonale, Bellinzona, Switzerland, and \\ ${ }^{3}$ Department of Nuclear Medicine, Marburg University Hospital, Marburg, Germany
}

Correspondence should be addressed to L Giovanella

Email

luca.giovanella@eoc.ch

\begin{abstract}
Objective: To assess the diagnostic performance of three high-sensitive assays in a cohort of TgAb-negative and TgAb-positive differentiated thyroid cancer (DTC) patients.

Design: Retrospective study on prospectively selected DTC patients.

Methods: Serum samples from 154 DTC patients were obtained 6-12 months after radioiodine ablation and tested by Beckman, Roche, BRAHMS Tg and TgAb assays, respectively. Receiver operating characteristics curves for Tg were plotted using outcome over time as benchmark and assay-specific Tg thresholds were obtained for TgAb-negative and TgAb-positive patients.

Results: The frequency of positive TgAb was 21, 20 and $20 \%$ for Beckman, Roche and BRAHMS, respectively. In TgAb-negative patients, clinical sensitivities and specificities of $100 \%$ and $85-95 \%$, respectively, were observed across all assays. In TgAb-positive patients, clinical sensitivities and specificities of $80-100 \%$ and $92-96 \%$, respectively, were observed using lower thresholds than in patients without TgAb.

Conclusions: Adopting appropriate thresholds, lower than those for TgAb-negative patients, is possible to reliably follow TgAb-positive patients using highly sensitive Tg assays.
\end{abstract}

\section{Introduction}

Serum thyroglobulin (Tg) measurements play a key role in the follow-up of patients with differentiated thyroid cancer (DTC) $(1,2)$. However, thyroglobulin autoantibodies (TgAb) can interfere with $\mathrm{Tg}$ measurements, potentially resulting in falsely low or undetectable results in widely employed $\mathrm{Tg}$ immunometric assays (Tg-IMA), (3, 4, 5). In earlier days, before the advent of reliable assays for the direct measurement of $\mathrm{TgAb}$ levels, the measurement of the recovery of added exogenous $\mathrm{Tg}$ (Tg-Rec) was employed to screen for TgAb interferences. This method however is strictly dependent on experimental conditions, has a relatively wide reference range and is not sufficiently

www.eje-online.org https://doi.org/10.1530/EJE-17-0663
() 2018 European Society of Endocrinology Printed in Great Britain
European Journal of

Endocrinology

(2018) 178, 75-82 sensitive and accurate for detecting assay interference with modern highly sensitive $\mathrm{Tg}$ assays $(3,4)$. In more recent years, so-called 'mini-recovery' tests (i.e. using a low additive $\mathrm{Tg}$ concentration) have been introduced as complementary test to $\operatorname{TgAb}$ measurement $(6,7)$ but have yet to be investigated extensively in patients with DTC. Finally, the measurement of Tg by liquid chromatography-tandem mass spectrometry (LC-MS/MS) recently emerged as a promising method to overcome antibody interferences in $\mathrm{Tg}$ measurement (8). In practice, however, many technical problems still affect $\mathrm{Tg}$ measurement by LC-MS/MS and, of note, the functional sensitivity (FS) of current methods (i.e. $\sim 0.5-1 \mu \mathrm{g} / \mathrm{L}$ ) 
results in a suboptimal clinical sensitivity. Accordingly, $\mathrm{Tg}$ measured with LC-MS/MS is undetectable in up to $40 \%$ of TgAb-positive patients having active disease (9). The direct measurement of TgAb using a sensitive immunometric method therefore remains the method of choice to screen for TgAb interferences. Tg results should be discarded in TgAb-positive patients, especially when $\mathrm{Tg}$ is rendered undetectable $(1,2,4,10)$. It should be taken into account, however, that (a) interference is variable between patients and Tg assays, (b) the degree of interference is only weakly correlated with $\mathrm{TgAb}$ concentrations and (c) TgAb detection is strongly method dependent $(1,2,3,4,5,6)$. Indeed, $\mathrm{TgAb}$ positivity does not indicate interference in itself and some cases with interference but apparently negative $\mathrm{TgAb}$ might be completely missed. For TgAb-positive patients, the use of serum levels of TgAb as a 'surrogate tumor marker' has been widely adopted in clinical practice (i.e. declining $\mathrm{TgAb}$ levels may indicate reduced tumor burden or the absence of disease while the persistence of anti-Tg antibodies, especially if levels are rising, may indicate persistent, recurrent or progressive thyroid cancer) $(1,3,4,11)$. However, the serum levels of TgAbs are not correlated with the tumor load of the patient, but rather indicate the activity of the immune system. Consequently, in some patients, there can be a change in $\operatorname{TgAb}$ status (negative to positive or vice versa) that is discordant with clinical status. Moreover, the eventual disappearance of $\mathrm{Tg}$ antibodies in median takes approximately $2-3$ years $(12,13)$. Interestingly, measurement of serum $\mathrm{Tg}$ by high-sensitive assays showed a good diagnostic accuracy even in patients carrying positive $\operatorname{TgAb}(14)$. Using DTC patient-derived serum pools, Giovanella and Ceriani compared the impact of $\mathrm{TgAb}$ interference on a conventional $\mathrm{Tg}$ assay with an FS of $0.9 \mu \mathrm{g} / \mathrm{L}$ and high-sensitive Tg assays with an FS of $0.1 \mu \mathrm{g} / \mathrm{L}$. They concluded that an undetectable Tg using the high-sensitive assay was unlikely to be a TgAb-induced false-negative result (15). McGrath et al. (16) compared conventional assays and a high-sensitive ELISA assay along TgAb status in a large sample series of DTC patients $(n=019)$. Among 392 TgAb-positive patients, $104(26 \%)$ were positive for $\mathrm{Tg}$ on the highsensitive but conventional assay, and in 65 of these patients $(62.5 \%)$, this corresponded to DTC recurrence. More recently, better sensitivity and specificity values were obtained in TgAb-positive DTC patients with high-sensitive $\mathrm{Tg}$ immunoassays (63-68\% and 71-77\%) compared to LC-MS/MS Tg measurement (56\% and $85 \%)$, respectively (17). Therefore, the present study was specifically undertaken to evaluate the performance of three commercially available automated high-sensitive Tg assays in monitoring TgAb-positive DTC patients and predicting their outcome over time.

\section{Subjects and methods}

\section{Institutional treatment and follow-up protocol}

At our Thyroid Centre, all DTC patients, except those with unifocal DTC $<1 \mathrm{~cm}$ in the largest diameter without extrathyroidal invasion, underwent (near-) total thyroidectomy with central or lateral neck dissection, depending on risk and intraoperative findings, subsequently followed by RRA in accordance with the European Association of Nuclear Medicine (EANM) recommendations (18). Most RRA treatments are performed 4 weeks after surgery without introducing T4 replacement (a TSH value $>30 \mathrm{IU} / \mathrm{L}$ is required in these cases), while rhTSH is employed in selected cases on indication of attending physician. Ablation activities between 1.1-3, 2-3.7 and 3.7-7.4 GBq are given in low-, intermediate- and high-risk patients as determined appropriate by the attending physician. Three to seven days after RRA, a post-treatment whole-body scan (PT-WBS) with combined integrated head and upper thorax single photon emission tomography/computed tomography (SPECT/CT) is performed following standard protocols as previously described (19). A PT-WBS showing physiological radioiodine distribution without uptake foci outside the thyroid bed (i.e. remnant) is rated as negative while a PT-WBS showing extra-thyroid non-physiological uptake foci is rated as positive. The latter findings are further clarified by neck US, fine-needle aspiration (FNA) and positron emission computed tomography/ CT (PET/CT), CT or magnetic resonance imaging (MRI), when indicated. Based on preoperative assessment, intraoperative findings, pathology reports, PT-WBS and additional imaging results patients are classified at high-, intermediate- or low-risk in accordance with the ATA 2015 guidelines (10). In low-to-intermediate risk and high-risk patients, TSH levels are maintained below 0.5 and $0.1 \mathrm{IU} / \mathrm{L}$, respectively, until cure is demonstrated and between $0.5-2.0$ and $0.1-1.0 \mathrm{IU} / \mathrm{L}$ later. The response to the initial treatment is evaluated 6-12 months after RRA using neck US and Tg testing. A diagnostic WBS is also performed in patients at intermediate and high risk and in those with positive TgAb. Primary treatment is considered completed if there is no evidence of residual 
disease; i.e. negative neck ultrasound and undetectable $\mathrm{Tg}$ (or negative WBS in TgAb-positive patients (4)). In other cases, further examinations are performed and additional treatments (i.e. surgery, radioiodine) are administered according to the judgment of the attending physician and multi-disciplinary thyroid oncological board.

\section{Study design}

All patients that underwent their early DTC posttreatment assessment in our center from 2005 to 2012 were retrieved from our database and standardized dataset (i.e, demographic data, surgical and pathological report, follow-up reports, whole-body scan and ultrasound reports and laboratory data) was obtained. Patients were selected for inclusion in the present study if (1) they underwent at least a (near-)total thyroidectomy resulting in apparent complete resection of neoplastic foci (R0); (2) they received I-131 for thyroid remnant ablation and the corresponding post-treatment whole-body scan (PT-WBS) showed no foci of I-131 uptake outside of the thyroid bed; (3) they received LT4 treatment and TSH levels were maintained below 0.1 and $0.5 \mathrm{UI} / \mathrm{L}$ (depending on the risk stratification) until the early follow-up visit (i.e. 6-12 months after ablation); (4) they underwent response assessment 6-12 months after ablation and corresponding residual serum samples (stored at $-80^{\circ} \mathrm{C}$ ) were available. The response to treatment was assessed as described earlier and rated as excellent response (ER), biochemical incomplete response (BIR), structural incomplete response (SIR) and indeterminate response (IR) according to the ATA 2015 guidelines (10). Follow-up visits consisted of a clinical examination with neck US and serum $\mathrm{Tg}$ and $\mathrm{TgAb}$ measurement under LT4 treatment on a yearly basis, with additional examinations performed on indication in selected cases. For each follow-up visit, a disease status was assigned by attending based on the longitudinal review of the available clinical, imaging, biochemical and cytological/histological data. Patients were classified as alive with no evidence of disease (NED) if there was no clinical, imaging or cytological/histological evidence of disease and their locally measured basal $\mathrm{Tg}$ and TgAb levels were undetectable or, if detectable, they were less than $1 \mathrm{ng} / \mathrm{mL}$ and had remained unchanged or declining over time ( $\mathrm{Tg}$ ) or had declined over time (TgAb). Patients who did not fulfill these criteria were classified as alive with evidence of disease (ED) and further stratified into alive with only biochemical evidence of disease (bED) or alive with structural evidence of disease recurrence $(\mathrm{sED})$, respectively. The disease-free survival (DFS) was calculated from the date of radioiodine ablation to the date of the last follow-up (NED patients) or the date of relapse detection (bREC and sREC patients), respectively.

\section{Laboratory measurements}

Once the inclusion for the study was completed, frozen sera aliquots were used to simultaneously measure $\mathrm{Tg}$ and $\operatorname{TgAb}$ levels on Elecsys e601 (Roche Diagnostics); UniCell DxI 800 (Beckmann Coulter, Fullerton, USA) and Kryptor (BRAHMS Gmbh, Henningsdorf, Germany) fullyautomated platforms according to the manufacturers' instructions (note: hereafter assays are referred to as Beckman, Roche and BRAHMS). Technical and analytical characteristics are summarized in Table 1 . TgAb values below functional sensitivity (FS) of any assay were considered negative. In the sense of this study, if $\mathrm{TgAb}$ was positive in one assay, this was assumed to only count for the corresponding Tg assay.

\section{Statistical analysis}

For statistical analysis, values of $\operatorname{Tg}$ ad $\operatorname{TgAb}$ below the FS were considered as equal to the FS of any method. The agreement between different assays was assessed by Passing and Bablok regression analysis. Categorical data were analyzed by chi-square testing or Z-test for proportions while continuous data were analyzed by parametric or non-parametric tests for differences in means or medians, respectively, depending on the normality of distribution. Continuous variables were dichotomized by receiver-operating characteristics (ROC) curve analysis

Table 1 Technical and analytical characteristics of $\mathrm{Tg}$ and TgAb assays.

\begin{tabular}{|c|c|c|c|c|}
\hline Assay & $\begin{array}{l}\text { Assay } \\
\text { principle }\end{array}$ & Assay format & FS & $\begin{array}{l}\text { Reference } \\
\text { standard }\end{array}$ \\
\hline \multicolumn{5}{|l|}{ Beckmann } \\
\hline $\mathrm{Tg}$ & ICMA & Direct & $0.1 \mu \mathrm{g} / \mathrm{L}$ & $\mathrm{BCR} \otimes 457$ \\
\hline $\mathrm{TgAb}$ & ICMA & Direct & $1.8 \mathrm{IU} / \mathrm{mL}$ & WHO 65/93 \\
\hline \multicolumn{5}{|l|}{ Roche } \\
\hline $\mathrm{Tg}$ & ECLIA & Direct & $0.1 \mu \mathrm{g} / \mathrm{L}$ & $\mathrm{BCR} \otimes 457$ \\
\hline $\operatorname{TgAb}$ & ECLIA & Competitive & $201 \mathrm{~J} / \mathrm{mL}$ & WHO 65/93 \\
\hline \multicolumn{5}{|l|}{ BRAHMS } \\
\hline $\mathrm{Tg}$ & TRACE & Direct & $0.15 \mu \mathrm{g} / \mathrm{L}$ & $\mathrm{BCR} \otimes 457$ \\
\hline $\operatorname{TgAb}$ & TRACE & Direct & $331 \mathrm{I} / \mathrm{mL}$ & WHO 65/93 \\
\hline
\end{tabular}

$B C R \circledast 457$, Certified Reference Material (European Commission Institute for Reference Materials and Measurements, Geel, Belgium); ECLIA, electrochemiluminometric immunoassay; FS, functional sensitivity; ICMA, immunochemiluminometric assay; TRACE, time-resolved amplified cryptate emission; WHO 65/93, World Health Organization International Standard. 
using the maximum value of Youden's index (J) as the most accurate cut-off point. The predictivity tests, i.e. sensitivity, specificity, positive (PPV) and negative (NPV) predictive value and accuracy, were calculated according to Galen and Gambino. DFS was estimated by using the Kaplan-Meier method, and differences between two curves were analyzed by log-rank or Mantel-Haenszel test and expressed as hazard radio (HR). Statistical significance was set at $P<0.05$. All statistical tests were performed by GraphPad Prism, version 7 (GraphPad Software).

\section{Ethics}

The protocol was approved by the Clinical Research Committee of Ente Ospedaliero Cantonale and the Ethics Committee of Canton Ticino (Bellinzona, Switzerland). All patients gave their informed consent before participating in the study.

\section{Results}

\section{Patients}

Two-hundred-fifty-six DTC patients who were assessed in for early post-ablation follow-up during the study period were retrieved. Seventeen patients (7\%) without a documented assessment of response to therapy and 85 (33\%) patients without available serum samples for laboratory studies were excluded. Thus, the final

Table 2 Patients' characteristics.

\begin{tabular}{lc}
\hline \multicolumn{1}{l}{ Characteristics } & Values \\
\cline { 1 - 2 } Gender & 154 \\
$\quad$ Females & $118(77 \%)$ \\
Males & $36(23 \%)$ \\
Age (years) & \\
$\quad$ Age at DTC diagnosis & $52 \pm 16.2(22-84)$ \\
Disease-free survival (months) & $36 ; 6-132$ \\
Overall series $(n=154)$ & $36 ; 24-132$ \\
NED $(n=123)$ & $32 ; 18-120$ \\
bED $(n=11)$ & $22 ; 6-96$ \\
SED ( $n=20)$ & \\
Histology & $135(88 \%)$ \\
PTC & $19(12 \%)$ \\
FTC & \\
Risk categories & $83(54 \%)$ \\
Low & $60(39 \%)$ \\
Intermediate & $11(7 \%)$ \\
High & \\
Radioiodine activity (GBq) & \\
1.1 & $46(30 \%)$ \\
2.0 & $69(45 \%)$ \\
3.7 & $39(25 \%)$ \\
\hline
\end{tabular}

study series comprised 154 (61\%) patients. Patient characteristics are summarized in Table 2. Median follow-up was 36 months (range 6-132 months). At the end of the study, twenty patients (13\%) had sED (15 patients with cervical lymph node metastases, 1 with neck and mediastinal lymph node metastases, 3 with lung metastasis, 1 with neck, mediastinum, lung and bone metastases) at a median time of 29 months (range 6-132) since RRA. These patients were initially treated by ${ }^{131}$ I therapy or additional surgery plus further course(s) of adjuvant ${ }^{131} \mathrm{I}$ therapy. Further additional treatments (i.e. external radiation therapy, thyrosin-kinase inhibitors) were administered on indication in selected cases. Eleven patients (7\%) had bED and were periodically monitored with neck US plus further additional imaging on indication. The remaining 123 patients (80\%) were disease-free (NED) until the last visit of follow-up (median time of 41.5 months (range 9-125)).

\section{Thyroglobulin antibodies}

Positive TgAb results were found in 33 (21\%), 32 (20\%) and 32 (20\%) of 154 DTC patients on Beckman, Roche and BRAHMS assay, respectively. Fifty-three patients (37\%) had positive $\mathrm{TgAb}$ in at least one assay, 28 (19\%) in two assays and $11(8 \%)$ in all assays, respectively $(P<0.001)$. No qualitative agreement was found between methods at regression analysis (data not shown) and $\mathrm{TgAb}$ concentrations differed significantly between different methods (Beckmann $1.80 \mathrm{IU} / \mathrm{mL} \quad$ (1.802260), Roche $20 \mathrm{IU} / \mathrm{mL}$ (20-3000), BRAHMS $33 \mathrm{IU} / \mathrm{mL}$ (33-1590), $P<0.0001$ ). As summarized in Table $3, \mathrm{TgAb}$ positivity rates and concentrations were similar in all patients' subgroups.

\section{Serum thyroglobulin in TgAb-negative and TgAb- positive patients}

The results of $\mathrm{Tg}$ measurements in TgAb-negative and TgAb-positive patients are summarized in Table 4 and Fig. 1. In all groups, Tg levels were significantly higher in SED and bED than NED patients, without significant differences between different assays.

\section{ROC curve analysis}

ROC curves were plotted to calculate the optimal $\mathrm{Tg}$ criterion to separate sED and NED patients in TgAbnegative and TgAb-positive groups (Fig. 2). Different $\mathrm{Tg}$ assays performed equally well and no differences 
Table 3 Serum TgAb in DTC patients.

\begin{tabular}{|c|c|c|c|c|}
\hline \multirow[b]{2}{*}{$\begin{array}{l}\text { Thyroglobulin } \\
\text { antibodies (TgAb) }\end{array}$} & \multicolumn{4}{|c|}{ Positivity rate $(n(\%))$} \\
\hline & Beckman & Roche & BRAHMS & $\begin{array}{l}\text { Chi-square } \\
(P)\end{array}$ \\
\hline $\operatorname{NED}(n=123)$ & $25(20 \%)$ & $25(20 \%)$ & $24(19 \%)$ & ns \\
\hline $\mathrm{bED}(n=11)$ & $3(27 \%)$ & $2(18 \%)$ & $2(18 \%)$ & ns \\
\hline $\operatorname{sED}(n=20)$ & $5(25 \%)$ & $5(25 \%)$ & $6(30 \%)$ & ns \\
\hline Chi-square $(P)$ & ns & ns & ns & \\
\hline
\end{tabular}

were found when the same $\mathrm{Tg}$ assay was employed in the presence or absence of $\mathrm{TgAb}$, respectively (Z-test for all AUCs' pairs, $P$ ns) (Table 5). Among TgAb-negative patients, assay-specific $\mathrm{Tg}$ thresholds were selected at $0.36,0.43$ and $0.31 \mu \mathrm{g} / \mathrm{L}$ for Beckman, Roche and BRAHMS, respectively. The corresponding sensitivity, specificity, accuracy and positive predictive value and negative predictive values obtained were 100, 92, 93, 65 and 100\% (Beckman); 100, 94, 95, 71 and 100\% (Roche) and 100, 85, 87, 87 and 100\% (BRAHMS), respectively. Positive and negative likelihood ratios (LR) were 10.0 and $0,16.3$ and 0 and 17.8 and 0.07 using Beckman, Roche and BRAHMS assays, respectively. Selected Tg thresholds were $0.20,0.12$ and $0.15 \mu \mathrm{g} / \mathrm{L}$ for Beckman, Roche and BRAHMS assays, respectively. The corresponding sensitivity, specificity, accuracy and positive predictive value and negative predictive value obtained were 80, 96, 92, 66 and 92\% (Beckman); 100, 92, 95, 71 and 100\% (Roche) and 100, 92, 93, 71 and $100 \%$ (BRAHMS), respectively. Positive and negative likelihood ratios (LR) were 20.0 and $0.21,12.5$ and 0 and 13.5 and 0 using Beckman, Roche and BRAHMS assay, respectively. Notably, independently from the presence or the absence of circulating TgAb, all sED patients were identified (excepting one TgAb-positive patient with local sED only missed by the Beckman Tg assay).
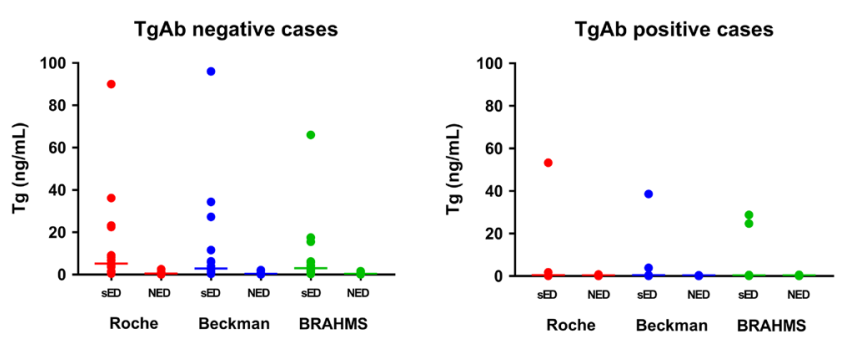

Figure 1

Serum Tg levels (expressed as median and range) in TgAb-negative and TgAb-positive DTC patients.

\begin{tabular}{|c|c|c|c|}
\hline \multicolumn{4}{|c|}{ Concentrations (IU/mL; median (range)) } \\
\hline Beckman & Roche & BRAHMS & Kruskal-WallisKrusk $(P)$ \\
\hline$\overline{1.80(1.80-2260)}$ & $\overline{20(20-3000)}$ & $33(33-1590)$ & $<0.0001$ \\
\hline $1.80(1.80-157)$ & $20(20-743)$ & $33(33-560)$ & $<0.0001$ \\
\hline $1.80(1.80-98)$ & $20(20-400)$ & $33(33-350)$ & $<0.0001$ \\
\hline ns & ss & ns & \\
\hline
\end{tabular}

\section{Prognostic role of serum Tg in TgAb- positive and -negative patients}

Differences in DFS according to Tg levels were assessed in TgAb-negative and TgAb-positive patients, respectively. Results are summarized in Fig. 3; briefly, serum Tg levels above the assay-specific Tg cutoffs predicted a significantly worse prognosis and a shorter DFS in both TbAb-negative and -positive patients' groups, respectively.

\section{Discussion}

The present study clearly shows that using high-sensitive Tg measurements, it is possible to predict outcome even in TgAb-positive patients, albeit using different thresholds than in patients without TgAb. This is of major importance for the aftercare of TgAb-positive patients, as thus far such patients could only be followed by means of serial $\mathrm{TgAb}$ measurements, amended by periodic neck ultrasound radioiodine scanning (4).

Although the present study certainly indicates that quantitative measurement of $\mathrm{Tg}$ levels in $\mathrm{TgAb}$-positive patients is still problematic in terms of quantitative accuracy, the loss of $\mathrm{Tg}$ due to $\mathrm{TgAb}$ interference in highly sensitive assays is not enough to drive Tg levels below the FS of the assays. This is in contrast with earlier Tg assay generations, where Tg levels could not be interpreted reliably with respect to the presence of remaining DTC tissue $(1,2,3,4,5)$. The current study clearly explains these earlier findings, as the cutoffs for all assays as identified by ROC-analysis, lies at levels below the FS of earlier assay generations. In addition, these differences might also be due to the use of a suboptimal number of capture or detection antibodies in older assays compared with the newer ones, which may have increased the likelihood that $\mathrm{TgAb}$ will hinder the binding of $\mathrm{Tg}$ to capture or detect antibodies (20). The present results are in line with a study reporting that the rate of undetectable Tg concentrations was about 2-fold lower for the 


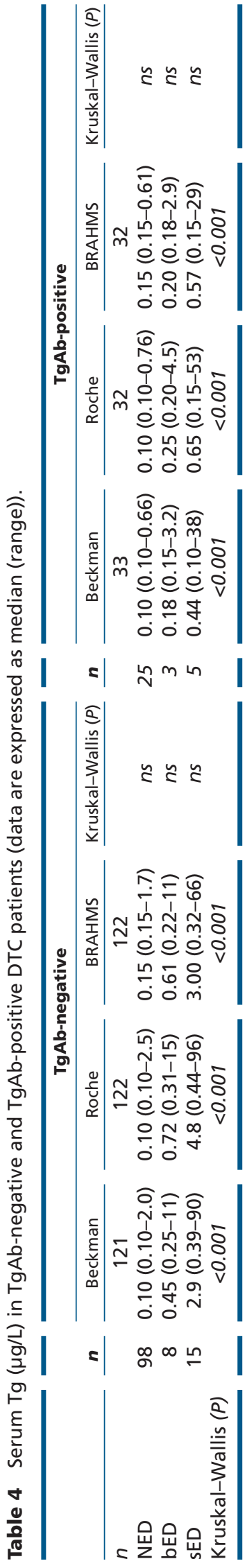

$\rightarrow$ Roche $\rightarrow$ Beckman $\rightarrow$ BRAHMS
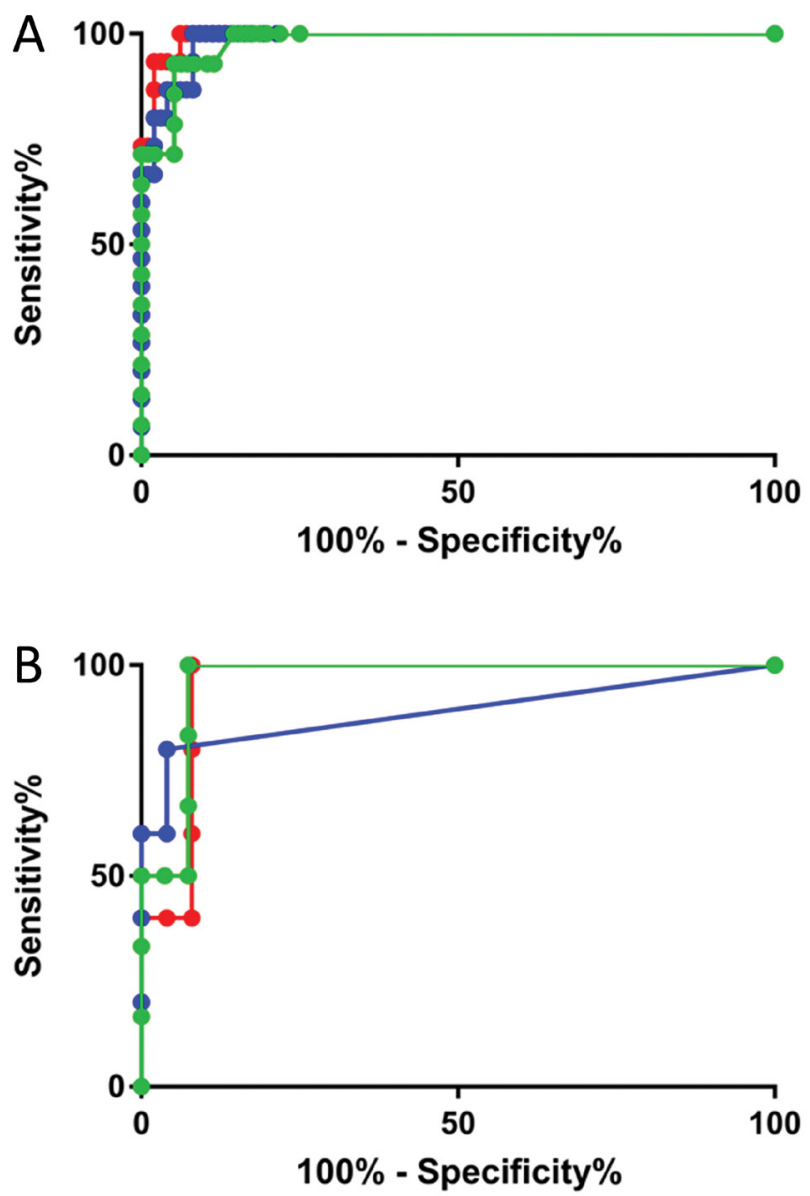

Figure 2

Serum $\mathrm{Tg}$ measured by different assays. ROC curve analysis in TgAb-negative (A) and TgAb-positive (B) patients.

high-sensitive Beckman, BRAHMS and Roche $\mathrm{Tg}$ immunoassays than for the conventional Siemens Immulite assay (17). In that study, however, the clinical sensitivity of high-sensitive Tg assays ranged from 53\% to $68 \%$, depending on the method, while in our present study, a better diagnostic performance was observed (clinical sensitivity 80-100\%). Differences in patient selection, disease status assignment and clinical endpoints at least partially account for these differences. Furthermore, Netzel et al. (17) selected their patient cohort retrospectively to have two numerically comparable groups of TgAb-positive and -negative patients; most of them were stage I papillary thyroid cancer free from disease at the time of blood draw (i.e. when the disease status was retrospectively assigned). Our data confirm the poor categorical and quantitative agreement between 
Table 5 Comparison of Tg ROC curves (data expressed as AUC/standard error (95\% confidence interval).

\begin{tabular}{l}
\hline \\
\hline TgAb-negative \\
TgAb-positive \\
Z-test $(P)$ \\
\hline
\end{tabular}

\begin{tabular}{c}
\hline Beckman \\
\hline $0.98 / 0.009(0.96-1)$ \\
$0.88 / 0.109(0.67-1.10)$ \\
$n s$
\end{tabular}

\begin{tabular}{c}
\hline Roche \\
\hline $0.99 / 0.006(0.98-1)$ \\
$0.95 / 0.038(0.87-1)$ \\
$n s$
\end{tabular}

\begin{tabular}{ccc}
\hline BRAHMS & & Z-test $(P)$ \\
\hline $0.97 / 0.012(0.95-1)$ & & $n s$ \\
$0.96 / 0.031(0.90-1)$ & & $n s$ \\
$n s$ & \\
\hline
\end{tabular}

different $\mathrm{TgAb}$ assays and, consequently, a significant proportion of cases with potential interferences could be missed - although it is conventionally assumed that $\operatorname{TgAb}$ assays and $\mathrm{Tg}$ assays from the same manufacturer are complimentary (4); this may not necessarily be the case. A major strength of our study is that we prospectively enrolled DTC patients (including high-risk ones) who were treated homogeneously in a tertiary referral center, and for each patient, obtained samples for Tg testing at the same time (6-12 months after ablation). With the available longitudinal follow-up at our center, it was possible to match results of $\mathrm{Tg}$ measurements against the outcome over time using structural recurrence as benchmark. Thus, our study design was able to better explain the merits of Tg assays, which also depend on pre-test probability. Some potential limitations should be addressed; first, while consecutive patients were enrolled and prospectively managed, the performance of $\mathrm{Tg} / \mathrm{TgAb}$ assays was evaluated by post hoc analysis in comparison to clinical outcome. However, these results were not taken into account in clinical management and, consequently, relevant biases are unlikely. Also, the period of postoperative follow-up of our patients might not be considered as long enough in at least part of the patient population. However, as in other large series from more recent years, the large majority of recurrences is encountered within $3-5$ years $(2,21)$ after diagnosis, we are still not likely to have missed many events. Furthermore, in spite of a low number of events, the results were still statistically significant, which can be considered an indicator that these significant results are also relevant for clinical practice. Conversely, any effects we may not have found are not very likely to be of a clinically relevant magnitude.

The present results can, if confirmed in further prospective studies, be of major influence on clinical practice. Thus far, patients with TgAb were considered difficult to follow as Tg assays could not be interpreted reliably. Based on the present results, it now however seems possible to interpret $\mathrm{Tg}$ assay results with respect to the risk of recurrence even in the presence of TgAb. This in turn would obviate the need to employ TgAb levels as a surrogate tumor marker. Instead the lower, but still detectable Tg levels can be followed by highsensitive Tg assay: an undetectable highly sensitive $\mathrm{Tg}$ measurement appears to indicate a complete remission of DTC regardless of the TgAb status. Furthermore, the need for repeated diagnostic whole-body scintigraphy purely for follow-up purposes would vanish; rather this modality would be reserved for post-ablation diagnostics if desired and for the localization of recurrence upon biochemical suspicion.

\section{Conclusion}

Adopting appropriate thresholds, lower than those for TgAb-negative patients, it is possible to reliably monitor TgAb-positive DTC patients using highly sensitive Tg assays.
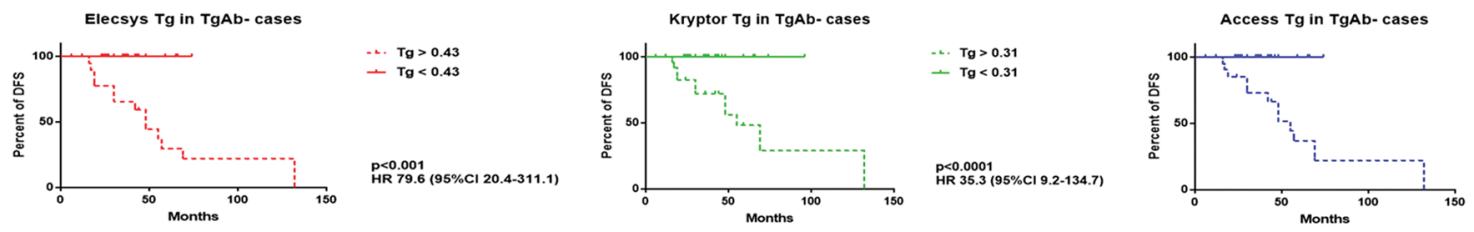

-.. $T g>0.36$
$-T g<0.36$

Elecsys $\mathrm{Tg}$ in $\mathrm{TgAb}+$ cases
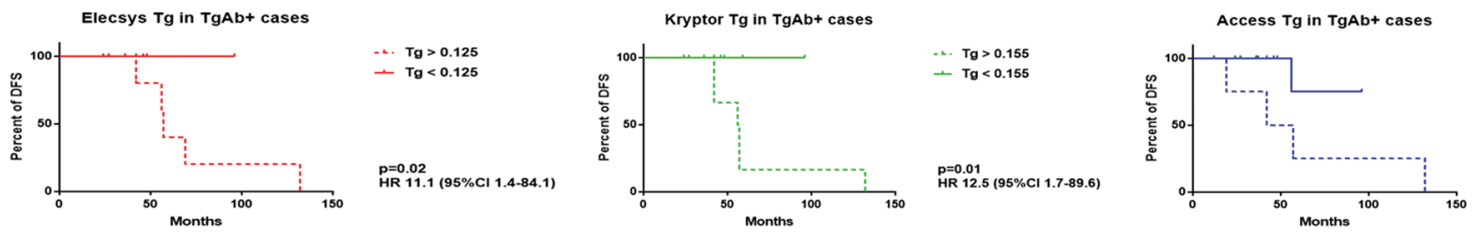

PR 49.1 (95\%Cl 12.9-186.5)

$\cdots T_{g}>0.20$
$-T_{g}<0.20$

$P=0.002$
HR $12.2(95 \% \mathrm{Cl} 1$ 1.2-115.8)

Figure 3

Kaplan-Meier curves of event-free survival in TgAb-positive and TgAb-negative patients according to Tg values below or above the most accurate ROC-derived cut-off levels. 


\section{Declaration of interest}

The authors declare that there is no conflict of interest that could be perceived as prejudicing the impartiality of the research reported.

\section{Funding}

This research did not receive any specific grant from any funding agency in the public, commercial or not-for-profit sector.

\section{References}

1 Spencer CA \& Fatemi S. Thyroglobulin antibody (TgAb) methods strenghts, pitfalls and clinical utility for monitoring TgAb positive patients with differentiated thyroid cancer. Best Practice and Research: Clinical Endocrinology and Metabolism 201327 701-712. (https://doi. org/10.1016/j.beem.2013.07.003)

2 Giovanella L, Clark PM, Chiovato L, Duntas L, Elisei R, FeldtRasmussen U, Leenhardt L, Luster M, Schalin-Jäntti C, Schott M et al. Thyroglobulin measurement using highly sensitive assays in patients with differentiated thyroid cancer: a clinical position paper. European Journal of Endocrinology 2014171 R33-R46. (https://doi.org/10.1530/ EJE-14-0148)

3 Spencer CA, Bergoglio LM, Kazarosyan M, Fatemi S \& Lopresti JS. Clinical impact of thyroglobulin (Tg) and Tg autoantibody method differences on the management of patients with differentiated thyroid carcinomas. Journal of Clinical Endocrinology and Metabolism 200590 5566-5575. (https://doi.org/10.1210/jc.2005-0671)

4 Verburg FA, Luster M, Cupini C, Chiovato L, Duntas L, Elisei R, Feldt-Rasmussen U, Rimmele H, Seregni E, Smit JW et al. Implications of thyroglobulin antibody positivity in patients with differentiated thyroid cancer: a clinical position statement. Thyroid $2013 \mathbf{1 0}$ 1211-1225. (https://doi.org/10.1089/thy.2012.0606)

5 Giovanella L, Feldt-Rasmussen U, Verburg FA, Grebe SK, Plebani M \& Clark PM. Thyroglobulin measurement by highly sensitive assays: focus on laboratory challenges. Clinical Chemistry and Laboratory Medicine 201553 1301-1314.

6 Verburg FA, Hartmann D, Grelle I, Giovanella L, Buck AK \& Reiners C. Relationship between antithyroglobulin autoantibodies and thyroglobulin recovery rates using different thyroglobulin concentrations in the recovery buffer. Hormone and Metabolic Research 201345 728-735. (https://doi.org/10.1055/s-0033-1349890)

7 Giovanella L, Imperiali M, Verburg FA \& Ceriani L. Evaluation of the BRAHMS Kryptor(®) thyroglobulin minirecovery test in patients with differentiated thyroid carcinoma. Clinical Chemistry and Laboratory Medicine 201351 449-453.

8 Hoofnagle AN, Becker JO, Wener MH \& Heinecke JW. Quantification of thyroglobulin, a low abundance serum protein, by immunoaffinity peptide enrichment and tandem mass spectrometry. Clinical Chemistry 200854 1796-1804. (https://doi.org/10.1373/ clinchem.2008.109652)

9 Azmat U, Porter K, Sentr L, Ringel MD \& Nabhan F. Thyroglobulin liquid chromatography-tandem mass spectrometry has a low sensitività for detecting structural disease in patients with antithyroglobulin antibodies. Thyroid 201727 74-80. (https://doi. org/10.1089/thy.2016.0210)

10 Haugen BR, Alexander EK, Bible KC, Doherty GM, Mandel SJ, Nikiforov YE, Pacini F, Randolph GW, Sawka AM, Schlumberger M et al. 2015 American Thyroid Association management guidelines for adult patients with thyroid nodules and differentiated thyroid cancer: the American Thyroid Association guidelines task force on thyroid nodules and differentiated thyroid cancer. Thyroid 201626 $1-133$.

11 Feldt-Rasmussen U, Verburg FA, Luster M, Cupini C, Chiovato L, Duntas L, Elisei R, Rimmele H, Seregni E, Smit JW et al. Thyroglobulin autoantibodies as surrogate biomarkers in the management of patients with differentiated thyroid carcinoma. Current Medicinal Chemistry 201432 3687-3692. (https://doi.org/10.2 174/0929867321666140826120844)

12 Chiovato L, Latrofa F, Braverman LE, Pacini F, Capezzone M, Masserini L, Grasso L \& Pinchera A. Disappearance of humoral thyroid autoimmunity after complete removal of thyroid antigens. Annals of Internal Medicine 2003139 346-351. (https://doi. org/10.7326/0003-4819-139-5_Part_1-200309020-00010)

13 Ringel MD \& Nabhan F. Approach to follow-up of the patient with differentiated thyroid cancer and positive anti-thyroglobulin antibodies. Journal of Clinical Endocrinology and Metabolism 201398 3104-3110. (https://doi.org/10.1210/jc.2013-1412)

14 Trimboli P, Zilioli V, Imperiali M \& Giovanella L. Thyroglobulin autoantibodies before radioiodine ablation predict differentiated thyroid cancer outcome. Clinical Chemistry and Laboratory Medicine 2017. (https://doi.org/10.1515/cclm-2017-0033)

15 Giovanella L \& Ceriani L. Comparison of thyroglobulin antibody interference in first- and second-generation thyroglobulin immunoassays. Clinical Chemistry and Laboratory Medicine 201149 1025-1027.

16 McGrath RT, Preda VA, Clifton-Bligh P, Robinson B, Sywak M, Delbridge L, Ward P, Clifton-Bligh RJ \& Learoyd DL. Is there a role for an ultrasensitive thyroglobulin assay in patients with serum antithyroglobulin antibodies? A large (Australian) cohort study in differentiated thyroid cancer. Clinical Endocrinology 201684 271-277. (https://doi.org/10.1111/cen.12736)

17 Netzel BC, Grebe SK, Carranza Leon BG, Castro MR, Clark PM, Hoofnagle AN, Spencer CA, Turcu AF \& Algeciras-Schimnich A. Thyroglobulin ( $\mathrm{Tg}$ ) testing revisited: $\mathrm{Tg}$ assays, $\mathrm{TgAb}$ assays and correlation of results with clinical outcomes. Journal of Clinical Endocrinology and Metabolism 2015100 E1074-E1083. (https://doi. org/10.1210/jc.2015-1967)

18 Luster M, Clarke SE, Dietlein M, Lassmann M, Lind P, Oyen WJ, Tennvall J \& Bombardieri E. European Association of Nuclear Medicine (EANM) Guidelines for radioiodine therapy of differentiated thyroid cancer.. European Journal of Nuclear Medicine and Molecular Imaging 200835 1941-1959. (https://doi.org/10.1007/ s00259-008-0883-1)

19 Giovanella L, Ceriani L, De Palma D, Suriano S, Castellani M \& Verburg FA. Relationship between serum thyroglobulin and 18FDGPET/CT in 131I-negative differentiated thyroid carcinomas. Head and Neck 201234 626-631. (https://doi.org/10.1002/hed.21791)

20 Marquet PY, Daver A, Sapin R, Bridgi B, Muratet JP, Hartmann DJ, Paolucci F \& Pau B. Highly sensitive immunoradiometric assay for serum thyroglobulin with minimal interference from autoantibodies. Clinical Chemistry 199642 258-262.

21 Verburg FA, Stokkel MP, Duren C, Verkooijen RB, Mader U, van Isselt JW, Marlowe RJ, Smit JW, Reiners C \& Luster M. No survival difference after (131)I ablation between patients with initially lowrisk and high-risk differentiated thyroid cancer. European Journal of Nuclear Medicine and Molecular Imaging 201037 276-283. (https://doi. org/10.1007/s00259-009-1315-6)

Received 14 August 2017

Revised version received 28 Sep 2017

Accepted 12 October 2017 